\title{
Formation of Pores during Unidirectional Solidification of Water Containing Carbon Dioxide *
}

\author{
Kenji Murakami and Hideo Nakajima \\ The Institute of Scientific and Industrial Research, Osaka University, Ibaraki 567-0047, Japan
}

\begin{abstract}
Water-carbon dioxide solutions of various concentrations of carbon dioxide are unidirectionally solidified upwards in a glass cell at various growth rates, and the formation of the pores of carbon dioxide is in-situ observed. Columnar pores are formed at low growth rates. The length of the columnar pores becomes shorter as the growth rate increases or as the carbon dioxide concentration increases. The distribution of the pores is not uniform but periodic along the solidification direction, except under the conditions of high growth rate and high concentration. The change in the length of the columnar pores and the periodic formation of the pores are explained in terms of the rate of supply of carbon dioxide to the growing pores from the surrounding liquid.
\end{abstract}

(Received May 13, 2002; Accepted August 20, 2002)

Keywords: water-carbon dioxide solution, unidirectional solidification, direct observation, pore formation, columnar pore, pore morphology, periodic formation of pores

\section{Introduction}

Much attention has been paid to the lotus-type porous metals that contain aligned long columnar pores. ${ }^{1-7)}$ Hyun et al. have shown that lotus-type porous copper has higher specific tensile strength when the tensile direction is parallel to the pore axis, compared with the conventional porous metals produced by, for example, foaming techniques or sintering methods. ${ }^{1,2)}$ Thus, lotus-type metals are promising both as structural materials and as functional materials. This new type of porous metal is fabricated by unidirectional solidification of molten metal dissolving hydrogen ${ }^{1-7)}$ or nitrogen..$^{8,9)}$ During solidification, the gas is rejected at the advancing solidification front due to the solubility gap between the solid and the liquid and forms columnar pores growing in the solidification direction together with the solid phase.

The formation of the gas pores (or blowholes) in castings and ingots has been extensively studied, ${ }^{10-13)}$ since the gas pores have been regarded as defects that degrade the mechanical properties of the metal products. For the purpose of studying the formation of gas pores, model experiments were widely carried out using water-gas systems and the nucleation and the growth of gas bubbles were directly observed in the course of solidification. Chalmers described the dependence of bubble formation on the freezing rate in waterair solution. ${ }^{14)}$ When the freezing is slow, more air diffuses into the bubbles from the surrounding water and they grow larger, while in the case where the growth rate is high there is less time for diffusion and the cross section of the bubbles decreases. Very slow freezing permits the air rejected at the ice/water interface to diffuse away from the interface and neither bubbles nor long columnar pores appear. It was reported $^{15,16)}$ that in the solidification of the water-air system and the water-carbon dioxide system in non-steady conditions, the shape of the gas pores, most of which are short pores rather than long columnar pores, depends on the growth rate of ice growing from the chill. In a unidirectional so-

*This Paper was Presented at the Spring Meeting of the Japan Institute of Metals, held in Narashino, on March 29, 2001. lidification of water dissolving air at controlled growth rates, Geguzin and Dzuba ${ }^{17)}$ showed that the air bubbles nucleated at the advancing solidification front grow into long columnar pores when the displacement rate of the pore cap is equal to the growth rate of the solid. They observed the periodic formation of elliptical pores along the solidification direction, which they attributed to the alternative accumulation and "drop down" of the liquid concentration near the solidification front.

For the purpose of simulating the formation of gas pores in lotus-type porous metals, it is preferable to unidirectionally solidify water-gas solutions at a constant rate and to use a gas having large solubility in water since the size and the pore density can be changed over a wide range by changing the concentration of the gas. In the present work, the formation of gas pores in water-carbon dioxide system solidifying at constant rates is directly observed to clarify the factors governing the morphology and the distribution of the gas pores.

\section{Experimental Procedure}

Water-carbon dioxide solution saturated with carbon dioxide was prepared by dissolving carbon dioxide in deaerated distilled water at $293 \mathrm{~K}$ and at atmospheric pressure. ${ }^{18)}$ This saturated solution was diluted with deaerated distilled water to obtain solutions with carbon dioxide concentrations of $117 \mathrm{~mm}^{3} / \mathrm{g}$ (degree of saturation $\left.=1 / 8\right), 234 \mathrm{~mm}^{3} / \mathrm{g}$ (degree of saturation $=1 / 4), 469 \mathrm{~mm}^{3} / \mathrm{g}($ degree of saturation $=1 / 2)$ and $937 \mathrm{~mm}^{3} / \mathrm{g}($ degree of saturation $=1)$ at $293 \mathrm{~K}$ and at atmospheric pressure. The solution was introduced into a glass cell $35 \mathrm{~mm}$ wide and $150 \mathrm{~mm}$ high. The thickness of the space of the glass cell was adjusted to about $160 \mu \mathrm{m}$.

The solution in the glass cell was solidified unidirectionally upwards by moving the glass cell down into an alcohol bath held at $253 \mathrm{~K}$ as shown in Fig. 1. The moving velocity of the glass cell was in the range from $3 \mu \mathrm{m} / \mathrm{s}$ to $45 \mu \mathrm{m} / \mathrm{s}$. As the solidification proceeded, the pores of carbon dioxide grew in the solidification direction together with ice. The experimental setup was placed in a plastic desiccator filled with flowing 

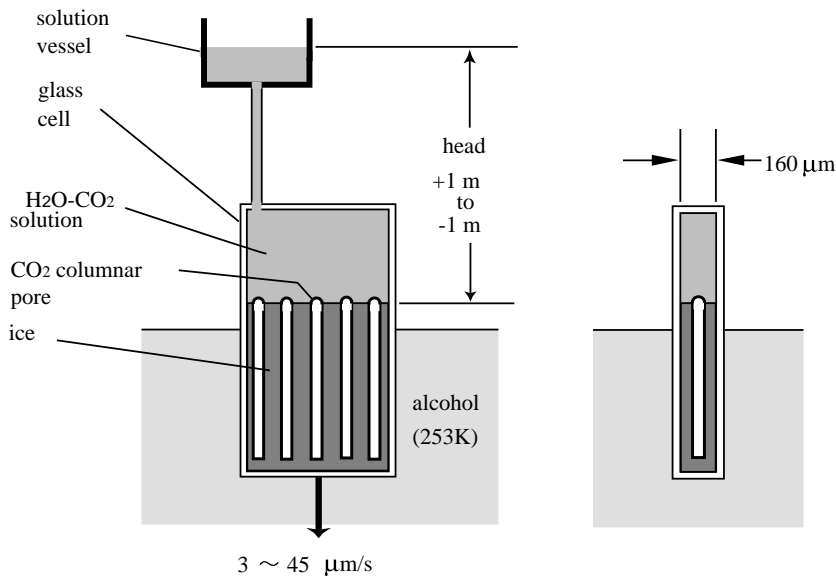

Fig. 1 Schematic drawing of the experimental setup.

nitrogen to keep the low temperature surface of the glass cell from being misted by humidity of the air. The nucleation process and growth process of the pores were observed directly by using a digital microscope through the glass window of the desiccator. The growth rate of ice was the same as that of the moving rate of the glass cell as long as the solidification front was observed under the microscope.

A thin flexible tube was connected to the top of the glass cell, and the other end of the tube was connected to a small vessel containing the water-carbon dioxide solution. In most of the experiments, the pressure at the solidification front was held at atmospheric pressure by keeping the level of the solution in the vessel at the level of the solidification front. The effect of the pressure on the pore growth was studied by changing the level of the solution in the vessel.

\section{Results}

The morphology of the carbon dioxide pores depends on the growth rate of ice as shown, for example, in Fig. 2 in the case of the solution with the degree of saturation of $1 / 2$. At the growth rate of $3 \mu \mathrm{m} / \mathrm{s}$, spherical pores that nucleated at the solidification front grow into long columnar pores elongated in the solidification direction as shown in Fig. 2(a). The solidification front is "planar" except that it is concave towards the solidification direction and that grain boundary grooves exist on the solidification front. As the growth rate increases, the length and the diameter of the columnar pores decrease and, at the growth rate of $12 \mu \mathrm{m} / \mathrm{s}$ and $18 \mu \mathrm{m} / \mathrm{s}$, only very short columnar pores or chestnut-shaped pores are formed as shown in Figs. 2(d) and (e). The morphology of the solidification front changes from planar one to cellular one with increasing growth rate due to the constitutional supercooling that occurs in the liquid adjacent to the solidification front. ${ }^{19)}$

Just after a pore has nucleated at the planar solidification front, very thin line extends in an instant downwards from the bottom of the pore as shown in Fig. 3(a). This line would be the grain boundary that was filled with liquid of high carbon dioxide concentration due to the grain boundary segregation during solidification, and would become visible because the carbon dioxide gas emerged and replaced the liquid. This observation indicates that the grain boundary grooves on the planar solidification front can be preferential sites for pore nucleation. ${ }^{20)}$ When the solidification proceeds with a cellular solidification front, the pores nucleate in the intercellular space just behind the cell tips, where the liquid is enriched in carbon dioxide. Immediately after the nucleation, a thin line filled with carbon dioxide extends from the bottom of the pore along the intercellular space as shown in Fig. 3(b). It is often observed that the thin line detached from the bottom of the pore directly after the formation of the line. This detachment is often accompanied by a quick and a short-distance movement of the nucleated pore in the upward direction. The columnar pore always grows upwards in the vertical direction even when the growth direction of the cells is not perpendicular to the solidification front, which occurs since the tips of the columnar pores are ahead of the cell tips and are, therefore, not affected by the cells.

Figure 4 summarizes the pore morphology as a function of the degree of saturation of the solution and the growth rate. The pore morphology mainly depends on the growth rate. At high growth rates, the pores nucleated at the solidification front are engulfed by the advancing solidification front, resulting in the formation of solidified region only with chestnut-shaped pores. When both the growth rate and the concentration of carbon dioxide are high, only a few chestnutshaped pores are formed. From the mass balance, carbon dioxide that is not incorporated in the pores should exist somewhere in the glass cell. It is observed that many nucleated pores detach from the solidification front and rise in the liquid ahead of the solidification front a few millimeters until they dissolve in the liquid ahead of the solidification front. The width of this liquid zone enriched in carbon dioxide will, however, be limited to no more than a few millimeters. In fact, neither the macroscopic variation of the solidification structure nor the pore morphology is observed along the solidification direction, which would be expected if macrosegregation occurred. The solubility of carbon dioxide in water increases with decreasing temperature ${ }^{18)}$ and, therefore, the carbon dioxide pores that rise in the liquid ahead of the solidification front, where the temperature is lower than $293 \mathrm{~K}$, dissolve in the liquid even when the initial solution is saturated with carbon dioxide at $293 \mathrm{~K}$. The condition of high carbon dioxide concentration and high growth rate is favorable for the formation of cells and the liquid in the intercellular space would be the possible second reservoir of carbon dioxide. In the case of the degree of saturation of $1 / 4$, the critical growth rate at which the pore morphology changes from columnar pore to short columnar pore and that at which the pore morphology changes from short columnar pore to chestnut-shaped pore hardly depend on the pressure at the solidification front in the pressure range from -1 to $1 \mathrm{mH}_{2} \mathrm{O}$ measured from the atmospheric pressure.

The distribution of the pores is not always uniform but periodic along the solidification direction depending on the conditions. The columnar pores stop growing one by one or some columnar pores simultaneously come to a halt during solidification, and a solidified zone is formed where the pore density is low. Numerous pores are, then, formed on the solidification front, which grow into columnar pores as the solidification further proceeds. An example of this periodic formation of pores is shown in Fig. 5 in the case of the degree of saturation of $1 / 4$ and the growth rate of $9 \mu \mathrm{m} / \mathrm{s}$. As shown in Fig. 6, the 


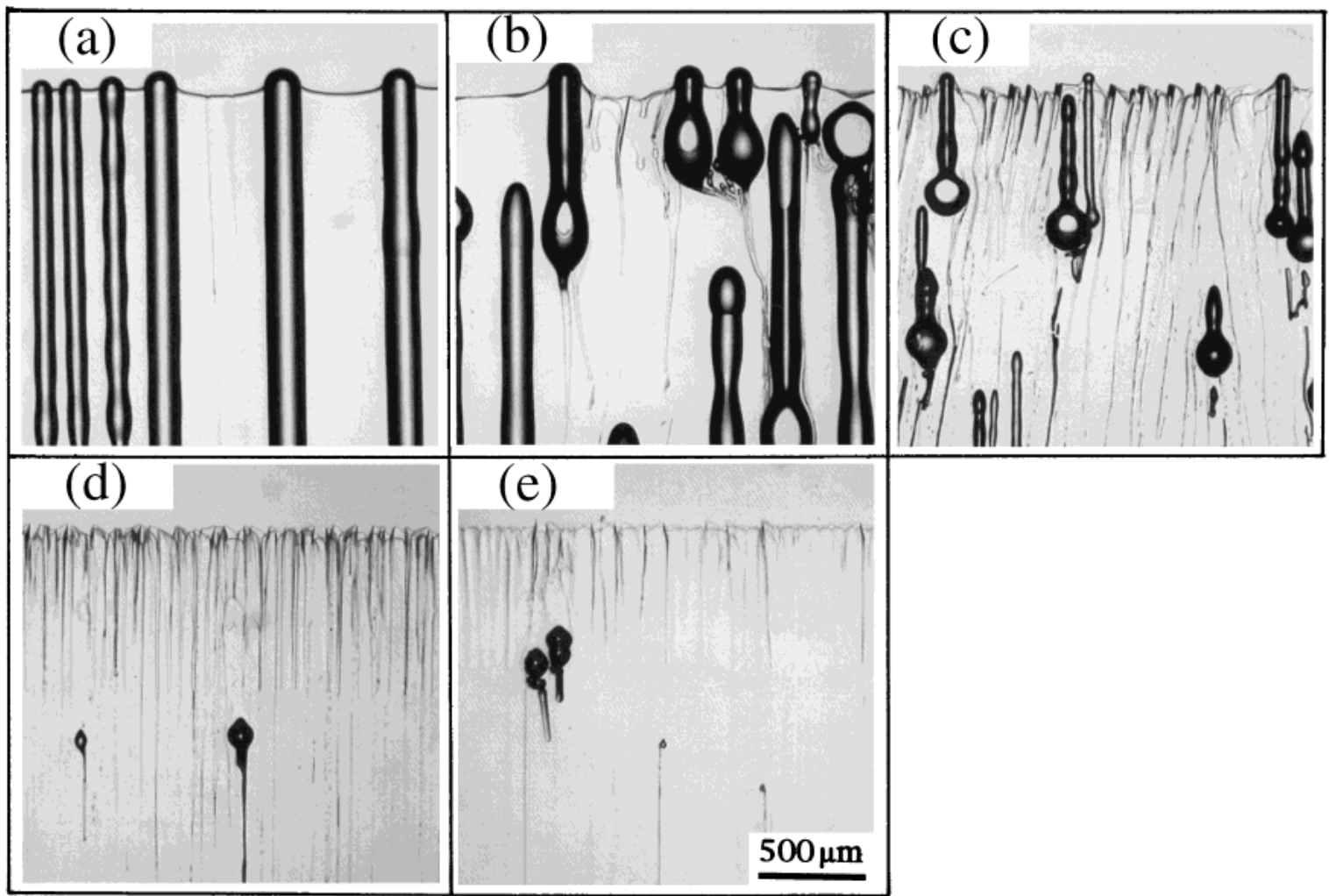

Fig. 2 Effect of the growth rate on the pore morphology. The degree of saturation of the solution is $1 / 2$. (a) $3 \mu \mathrm{m} / \mathrm{s}$, (b) $6 \mu \mathrm{m} / \mathrm{s}$, (c) $9 \mu \mathrm{m} / \mathrm{s}$, (d) $12 \mu \mathrm{m} / \mathrm{s}$ and (e) $18 \mu \mathrm{m} / \mathrm{s}$.

periodicity appears at low growth rates or for low degrees of saturation. The region in the figure denoted by "(periodic)" is a transition region from "periodic" to "aperiodic", where the periodic formation of pores is not so clear.

The periodic distance decreases with increasing growth rate and hardly depends on the degree of saturation of the solution within the experimental conditions in the present work as shown in Fig. 7. At the growth rate of $3 \mu \mathrm{m} / \mathrm{s}$, although the periodic formation of pores was ascertained with unaided eye, the periodic distance could not be measured since the distance was larger than the width of the solidification zone where the pores could be observed with a digital microscope. The pressure in the glass cell has little effect on the periodic distance in the pressure range from -1 to $1 \mathrm{mH}_{2} \mathrm{O}$ measured from the atmospheric pressure when the degree of saturation is $1 / 4$.

Figure 8 shows the diameter of columnar pores that nucleate and grow under a same applied pressure for four different degrees of saturation. The diameter decreases with increasing growth rate and with decreasing degree of saturation. When the solution with the degree of saturation of unity is solidified at a growth rate of $3 \mu \mathrm{m} / \mathrm{s}$, the pore diameter is larger than the width of the space of the glass cell. The cross section of this pore is plate-like and is interposed between the two glass plates. The increase in the applied pressure from -1 to $1 \mathrm{mH}_{2} \mathrm{O}$ results in the decrease in the pore diameter by about $25 \mu \mathrm{m}$ in the case of the degree of saturation of $1 / 4$ and at growth rates ranging from 3 to $9 \mu \mathrm{m} / \mathrm{s}$.

The pore diameter is sensitive to the applied pressure. When the applied pressure is suddenly decreased and then held at the pressure, the pore diameter temporarily becomes large and then decreases to a diameter that is slightly larger than the original value before the reduction in pressure. A larger diameter can be maintained by continuously decreasing the pressure as Fig. 9(a) shows, where the decreasing rate of the pressure is $2 \times 10^{-3} \mathrm{mH}_{2} \mathrm{O} / \mathrm{s}$. On slightly increasing the pressure, the pore diameter becomes small. If the pressure increase is large, the pore cap shrinks and the solid encircling the pore cap starts to cover it. Finally the columnar pore is engulfed by the advancing solidification front as shown in Fig. 9(b). The diameter of the pore can be periodically changed by changing the pressure periodically (Fig. 9(c)). The pictures in Fig. 9 were taken in this order: Figs. 9(a), (b) and (c). After the picture in Fig. 9(a) has been taken, the applied pressure was increased. The columnar pore at the right end in Fig. 9(b) survived this pressure increase, and the effect of the periodic pressure change was examined during the subsequent growth of this columnar pore.

Some examples of the interactions between the pores are shown in Figs. 10 and 11. When the tip of the columnar pore (A) in Fig. 10 was at the solidification front, the pore (B) nucleated near the columnar pore (A), which caused the cessation of the columnar pore (A). The nucleation of the pore (C) at the solidification front did not stop the growth of the columnar pore (D) but reduced the diameter of the latter, and the columnar pore (D) thickened after the columnar pore (C) has come to a halt. The growth directions of the columnar pores (A) and (B) in Fig. 11 make a small angle. The tips of these two columnar pores coalesced in an instant and one columnar pore grows thereafter. 

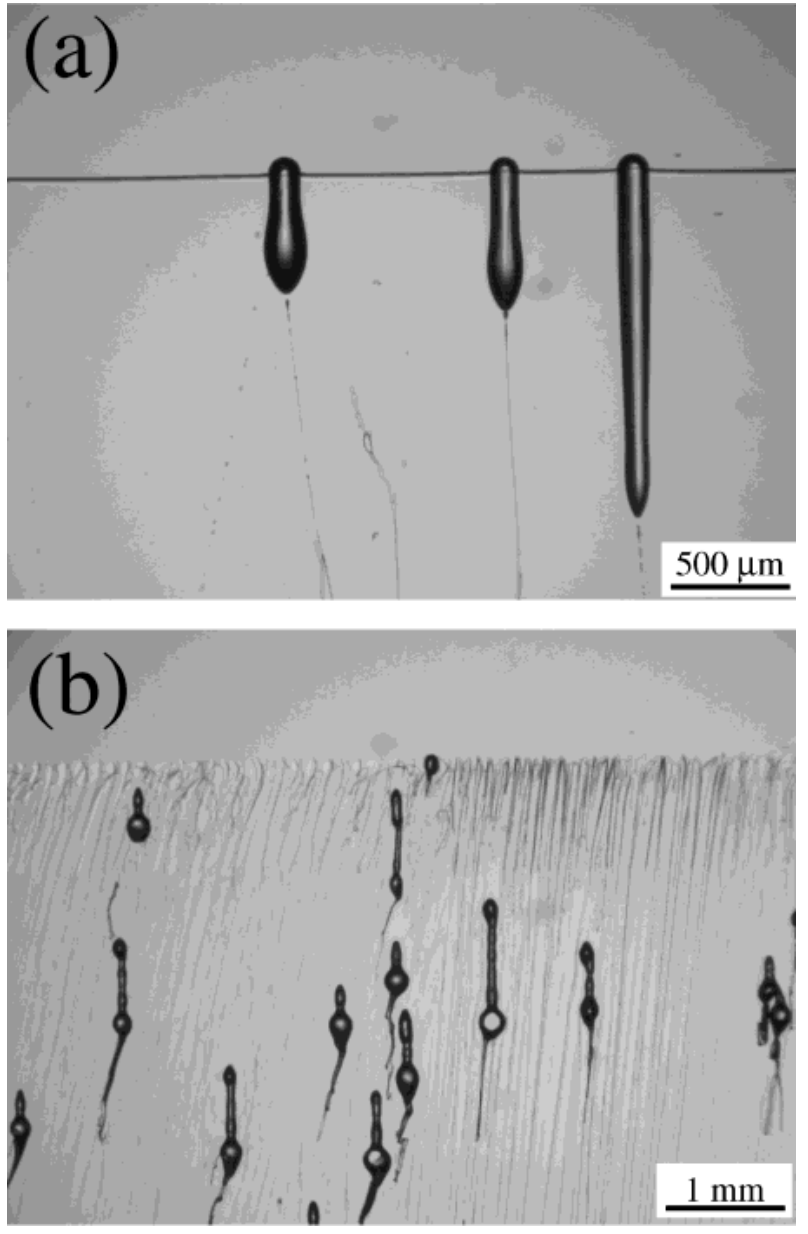

Fig. 3 Thin lines extending from the bottom of the pores. (a) The degree of saturation of the solution is $1 / 4$ and the growth rate is $3 \mu \mathrm{m} / \mathrm{s}$, (b) the degree of saturation is unity and the growth rate is $9 \mu \mathrm{m} / \mathrm{s}$.

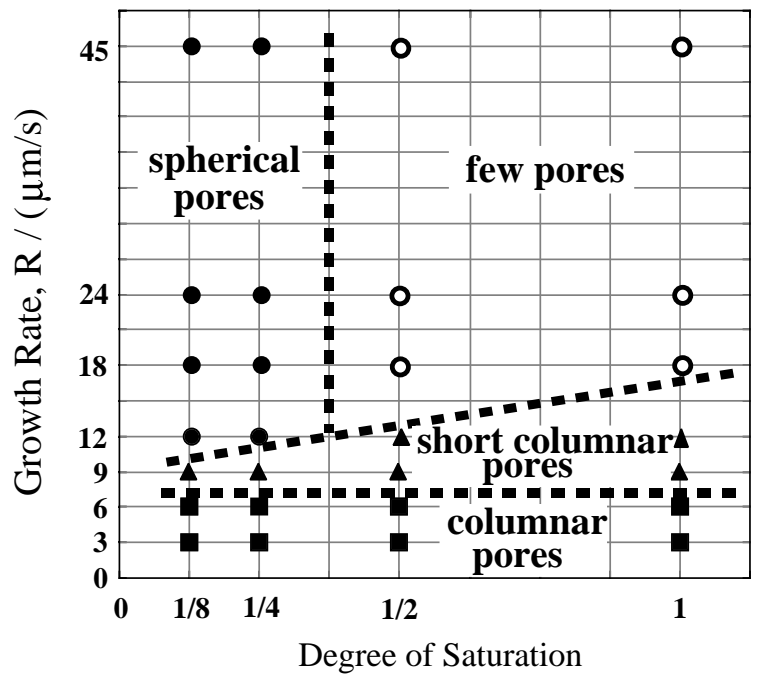

Fig. 4 Morphology of the pores as a function of the degree of saturation and the growth rate.

\section{Discussions}

During solidification, the carbon dioxide dissolved in the liquid is partially rejected at the advancing solidification front, since the equilibrium distribution coefficient of carbon dioxide at the solid/liquid interface is less than unity. ${ }^{16)}$ The pores

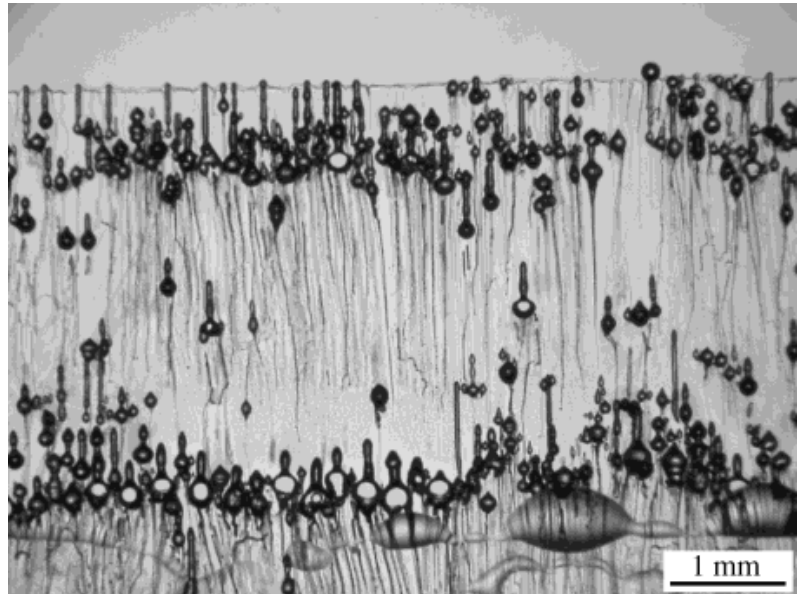

Fig. 5 Periodic formation of pores. The degree of saturation of the solution is $1 / 4$ and the growth rate is $9 \mu \mathrm{m} / \mathrm{s}$.

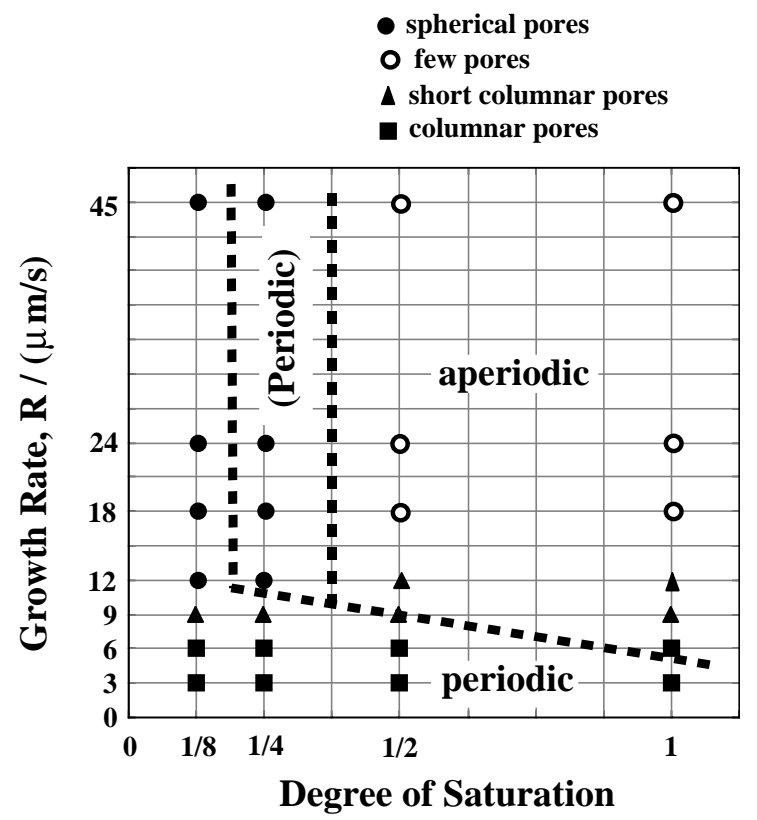

Fig. 6 Dependence of the periodicity of pore formation on the degree of saturation of the solution and the growth rate.

of carbon dioxide nucleate when the concentration at the solidification front reaches a critical value. The nucleated pores incorporate carbon dioxide from the surrounding liquid and grow into columnar pores.

There may be two factors that shorten the length of columnar pores as the growth rate increases. The amount of carbon dioxide to be incorporated in the pore should be balanced with that supplied from the surrounding liquid by diffusion to maintain the growth of columnar pores. As the growth rate increases, the rate of supply becomes insufficient for the pore elongation and the tips of the columnar pores are engulfed by the growing solid. The second factor is related with the morphology change of the solidification front with the growth rate. Planar solidification front is realized at slower growth rates, and the concentration of the liquid at this front that advances at a constant rate is approximated by the concentration of the bulk liquid divided by the equilibrium distribution coefficient of carbon dioxide that is reported to be $0.275 .{ }^{16)}$ On the 


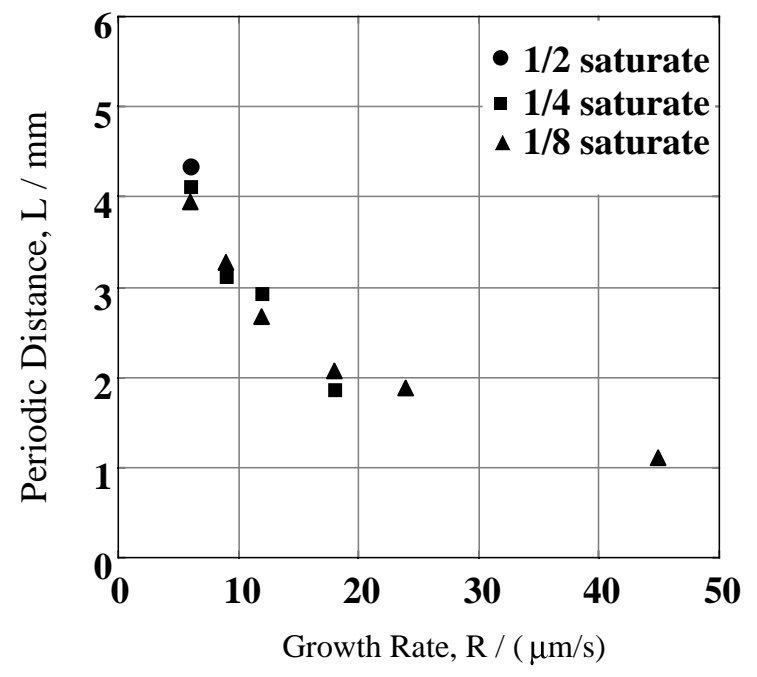

Fig. 7 Periodic distance for pore formation against the degree of saturation of the solution and the growth rate.

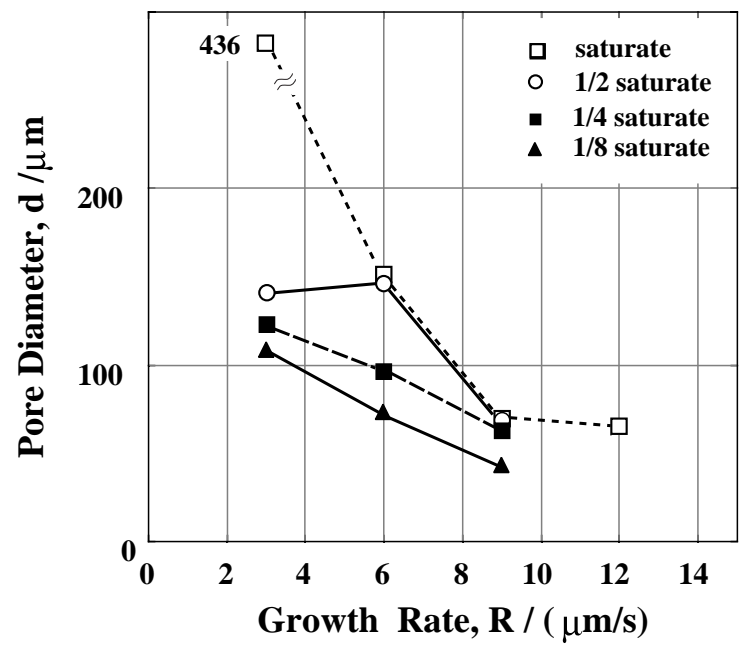

Fig. 8 Pore diameter against the degree of saturation of the solution and the growth rate.

other hand, the solidification front is cellular when the growth rate is high, and the concentration of the liquid at the solidification front is closer to that of the bulk liquid due to the curvature effect of the cell tip. ${ }^{21)}$ The concentration of the liquid at the solidification front in the latter case is lower than that in the former case. The supply of carbon dioxide will, therefore, be insufficient and the growth of long columnar pores will be hampered as the growth rate increases.

The carbon dioxide in the liquid around the pore cap is incorporated in the pores and the liquid will be depleted in carbon dioxide unless the steady state solidification is strictly realized, resulting in the cessation of the pore growth. As the solidification front further advances, carbon dioxide rejected at the front will gradually accumulate in the liquid near the front. When the concentration of carbon dioxide reaches a critical value, new pores will nucleate and grow. As a result of these depletion and accumulation of carbon dioxide, the periodicity of pore distribution appears.

In unidirectional solidification of a single phase alloy with a planar solidification front, the solute concentration $C_{\mathrm{L}}^{*}$ in the liquid at the solidification front increases as the solidifi-

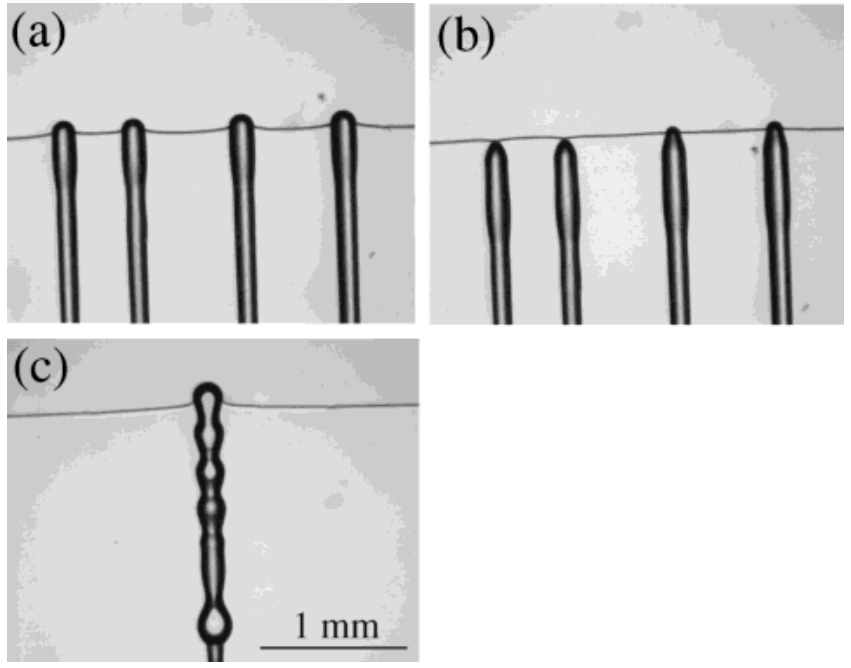

Fig. 9 Effect of the applied pressure on the growth of columnar pores. The pressure is (a) decreasing, (b) increasing and (c) periodically changing.

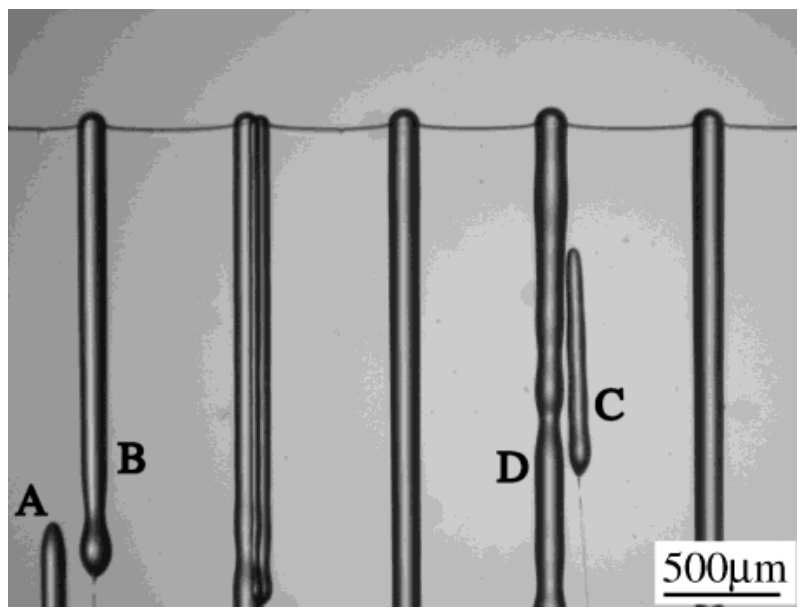

Fig. 10 Interaction between pores.

cation proceeds in the initial transient region. $C_{\mathrm{L}}^{*}$ is given as follows, ${ }^{22)}$

$$
C_{\mathrm{L}}^{*}=\frac{C_{0}}{k}\left[1-(1-k) \exp \left(-\frac{k R x}{D}\right)\right]
$$

where $C_{0}$ : initial solute concentration

$k$ : equilibrium distribution coefficient of solute

$R$ : growth rate

$x$ : distance from the mold wall

$D$ : diffusion coefficient of solute in the liquid.

In the case that a zone having very low pore density is periodically formed as shown in Fig. 5, $x$ is the distance measured from the position where the zone starts. It is known from the above equation that the distance for $C_{\mathrm{L}}^{*}$ to reach a specific value, which in the present work is the critical concentration for pore nucleation, decreases with increasing $R$. The morphology of the solidification front changes from planar morphology to cellular one with increasing growth rate and, in this case, the critical distance will also depend on the morphology of the solidification front. A rigorous analysis on the solute concentration in the intercellular space in the initial transient as well as studies on the nucleation site of pores will be necessary to quantitatively explain the dependence of the 


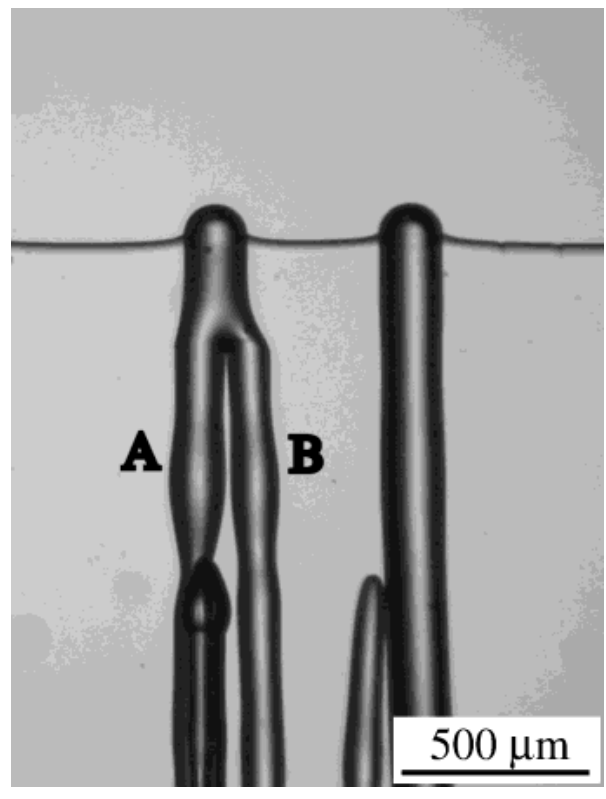

Fig. 11 Coalescence of columnar pores.

periodic distance on the growth rate shown in Fig. 7.

The columnar pore has a large volume filled with carbon dioxide gas behind the solidification front. When the applied pressure in the glass cell is suddenly reduced a little and then held at the pressure, this gas expands and the tip of the columnar pore becomes large. In order to maintain the growth of the columnar pore having this large diameter, more carbon dioxide than that supplied to the pore before the reduction in the applied pressure is needed. The supply of carbon dioxide, however, is limited by the diffusion from the surrounding liquid to the pore. Accordingly, the pore diameter decreases until the supply and the demand of carbon dioxide balance each other. This final diameter is slightly larger than the original value since the pressure in the columnar pore is a little lower than that before the reduction in the applied pressure. A columnar pore having constant larger diameter can be obtained by continuously decreasing the applied pressure as shown in Fig. 8(a).

The pore diameter and the pore spacing will depend on the diffusion of carbon dioxide in the liquid. In the present work, although the advancing rate of the solidification front is constant during an experimental run as long as the solidification front is observed under a microscope, the columnar pores stop growing one by one or some columnar pores simultaneously come to a halt. The pore spacing, therefore, could not be measured as a function of growth rate. The carbon dioxide to be incorporated in the growing pores diffuses from the liquid around the pore tips. As the advancing rate of the solidification front increases, the distance over which carbon dioxide can diffuse becomes short. As a result, the volume of the above mentioned liquid becomes small, leading to the reduction of the pore volume and the pore diameter.

When a solution with the degree of saturation of $1 / 4$ is solidifying with a planar solidification front in a steady state, the concentration of the liquid at the front is given by the bulk concentration divided by the equilibrium distribution coefficient and is about $800 \mathrm{~mm}^{3} / \mathrm{g}$ at $273 \mathrm{~K}$. Accordingly, for the nucleation of a pore at the solidification front, carbon diox- ide dissolved in the liquid having a volume roughly equal to that of the pore is necessary. The liquid around the nucleated pore is, hence, depleted in carbon dioxide and the growth of a columnar pore growing nearby comes to a halt or its diameter becomes small. On the contrary, if one of the two columnar pores growing close to each other stops growing, the diameter of the other becomes large since more carbon dioxide diffuses into this columnar pore.

Most of the experimental results in the present work can be understood in terms of the rejection of carbon dioxide at the solidification front and its diffusion in the solution. Principal factors controlling the pore formation will be common to the water-carbon dioxide system and the metal-gas system. Direct observation of pore nucleation and growth in the watercarbon dioxide system may give us information on the process parameters to control for the fabrication of porous metals with desired morphology, size and distribution of pores.

\section{Conclusions}

Water-carbon dioxide solutions of various concentrations of carbon dioxide are unidirectionally solidified at various growth rates in a glass cell and the formation of carbon dioxide pores is directly observed. The results obtained are as follows:

(1) Columnar pores are formed at low growth rates. The length of the columnar pores decreases with increasing growth rate or concentration of carbon dioxide in the solution.

(2) The solidification front is planar when both the growth rate and the concentration of carbon dioxide are low. The pores nucleate preferentially at the grain boundary grooves on the solidification front. On increasing growth rate or concentration of carbon dioxide, the solidification front becomes cellular. In this case, the pores nucleate in the intercellular region just behind the tips of the cells.

(3) The distribution of the pores is periodic along the solidification direction at low growth rates or for low concentrations of carbon dioxide. The periodic distance decreases with increasing growth rate.

(4) In the case that both the nucleation and the growth of the pores occur at a same applied pressure, the diameter of the columnar pore increases with decreasing pressure. On the other hand, when the applied pressure is suddenly reduced a little and held at the pressure, the pore diameter temporarily becomes large and then decreases to a value that is slightly larger than the original value.

\section{Acknowledgments}

The authors are grateful to Mr. Y. Nakai, an expert technical staff of The Institute of Scientific and Industrial Research, Osaka University, for manufacturing a part of the experimental setup.

\section{REFERENCES}

1) H. Nakajima, S. K. Hyun, K. Ohashi, K. Ota and K. Murakami: Colloids Surf. 179 (2001) 209-214.

2) S. K. Hyun, K. Murakami and H. Nakajima: Mater. Sci. Eng. A299 
(2001) 241-248.

3) S. Yamamura, H. Shiota, K. Murakami and H. Nakajima: Mater. Sci. Eng. A318 (2001) 137-143.

4) S. K. Hyun, Y. Shiota, K. Murakami and H. Nakajima: Proc. Int. Conf. Solid-Solid Phase Transformations'99 (JIMIC-3), Kyoto, Japan, May 24-28, (1999) pp. 341-344.

5) H. Nakajima, S. K. Hyun, K. Ohashi, K. Ota and K. Murakami: Proc. 3rd Int. Conf. High Pressure School, Warsaw, Poland, Sept. 13-16, (1999) pp. 37-40.

6) L. V. Boiko, V. I. Shapvalov, E. A. Chernykh, I. A. Rastorgueva, V. P. Martynov and V. I.Dolzhenkov: Poroshkovaya Metallurgiya 346[10] (1991) 78-81.

7) A. E. Simone and L. J. Gibson: Acta Mater. 44 (1996) 1437-1447.

8) S. K. Hyun and H. Nakajima: Proc. Int. Conf. Cellular Metals and Metal Foaming Technol., Bremen, Germany, Jun. 18-20, (2001) pp. 181-186.

9) S. K. Hyun and H. Nakajima: Mater. Trans. 43 (2002) 526-531.

10) Metals Handbook, 9th edition, Vol. 15, Casting, (ASM International, Metals Park, O., 1998) pp. 82-87.

11) M. C. Flemings: Solidification Processing, (McGraw-Hill, New York,
1974) pp. 208-210.

12) J. Campbell: Castings, (Butterworth-Heinemann, Oxford, 1998) pp. $162-173$.

13) H. Fredriksson and I. Svensson: Metall. Trans. 7B (1976) 599-606.

14) B. Chalmers: Sci. Am. 200 (1959) 114-122.

15) P. S. Wei, Y. K. Kuo, S. H. Chiu and C. Y. Ho: Int. J. Heat and Mass Transfer 43 (2000) 263-280.

16) K. F. Vasconcellos and J. Beech: J. Cryst. Growth 28 (1975) 85-92.

17) Ya. E. Geguzin and A. S. Dzuba: J. Cryst. Growth 52 (1981) 337-344.

18) Handbook of Chemistry, 3rd edition, Pure Chemistry II, edited by Chem. Soc. Japan, (Maruzen, Tokyo, 1984) p. 158.

19) B. Chalmers: Principles of Solidification, (John Wiley \& Sons, New York, 1964) pp. 150-163.

20) J. C. Fisher: J. Appl. Phys. 19 (1948) 1062-1067.

21) W. Kurz and D. J. Fisher: Fundamentals of Solidification, 3rd edition, (Trans Tec Publications, Switzerland, 1989) pp. 63-71.

22) M. C. Flemings: Solidification Processing, (McGraw-Hill, New York, 1974) pp. 36-41. 